\title{
Controller Design Using Laguerre Functions
}

\author{
Philip D Olivier
}

March 5, 2021

\begin{abstract}
This paper was prepared for presentation at the Utah Academy of Sciences, Arts and Letters March 20, 2021 meeting.

This paper presents a technique for designing controllers for rational plants using Laguerre functions. The inputs to the process are the a) the plant transfer function $\mathrm{P}(\mathrm{s}), \mathrm{b})$ the target transfer function $\mathrm{T}(\mathrm{s})$ and $\mathrm{c}$ ) the desired order of the controller. Future work will extend this process so it is applicable to 1) irrational continuous time plants and 2) to irrational discrete time plants.
\end{abstract}

\section{Introduction}

This paper begins by stating the traditional controller design problem for continuous time systems described by Laplace transform transfer functions. It then quickly reviews the Laplace domain Laguerre functions. This paper uses this machinery to derive controller design equations of the form

$$
\left[\begin{array}{ll}
-D & N
\end{array}\right] p=0, \quad p=C \hat{p}+b
$$

where the matrices $N$ and $D$ are generated by coefficients of Laguerre expansions of known transfer functions, $p$ is the column vector of parameters of the controller, and $\hat{p}$ is a reduced parameter vector that satisfies the constraint equation $p=C \hat{p}+b$ that does one or more things: a) it guarantees that the design equations have a nontrivial solution by guaranteeing that the denominator of the controller transfer function is monic; and b) it might impose other constraints.

The solution to (1) is

$$
\hat{p}=-\left(\left[\begin{array}{ll}
-D & N
\end{array}\right] C\right)^{+}\left[\begin{array}{ll}
-D & N
\end{array}\right] b \quad p=C \hat{p}+b
$$

where $M^{+}$is the pseudo inverse of $\mathrm{M}$ and is easily obtained in Matlab.

\subsection{Controller Design Problem}

Given 1) a good model of a plant, $P(s)$, and 2) design specifications for the closed loop system, design (or choose) a controller with transfer function $C(s)$, 
so that the closed loop system meets the design specifications in some sense. Once the controller is designed, the closed loop transfer functions are $T_{1}(s)=$ $P(s) C(s) /[1+P(s) C(s)]$, or $T_{2}(s)=P(s) /[1+P(s) C(s)]$, or $T_{3}(s)=1 /[1+$ $P(s) C(s)]$. A partial list of common design specifications might be

1. closed loop stability

2. disturbance rejection

3. noise rejection

4. input tracking

Once $C(s)$ is chosen, it is expected that $T(s)$ exhibits the desired characteristics. There is a very rich literature on how to design controllers to achieve each of these objectives, as well as many more. This paper takes a different approach. It first chooses, or designs, target closed loop transfer functions, $T_{i}(s)$ that has the desired properties and then finds the controller transfer function, $C(s)$ that produces the desired $T_{i}(s) \mathrm{s}$. To keep the development simple, only one target transfer function will be considered, i.e. $T_{1}(s)=T(s)$.

\subsection{Laguerre Functions}

For background on Laguerre Functions, the reader is referred to [1] which also includes many applications. Some of the author's earlier work with Laguerre Functions can be found in [2] and [3]. The Laguerre functions span an inner product space in the s-domain with inner product

$$
\langle F(s), G(s)\rangle=\frac{1}{2 \pi j} \oint_{\Gamma} F(s) G(-s) d s
$$

where the contour $\Gamma$ encloses the entire right half plane in the counter clockwise direction. The Laguerre functions have the form

$$
L_{k}(s)=\sqrt{2 p} \frac{(s-p)^{k}}{(s+p)^{k+1}}, \quad 0<p=\operatorname{Re}(p), \quad k=0,1,2, \ldots
$$

The Laguerre functions are orthonormal

$$
\left\langle L_{i}(s), L_{k}(s)\right\rangle=\delta_{i, k}
$$

Well behaved transfer functions can be expanded in Laguerre functions

$$
T(s)=\sum_{k=0}^{\infty} t_{k} L_{k}(s) \quad t_{k}=\left\langle T(s), L_{k}(s)\right\rangle
$$

When $G(s)$ has poles only in the LHP (left half plane), a convenient way to compute the inner product of $G(s)$ and $L_{k}(s)$ is

$$
\left\langle G(s), L_{k}(s)\right\rangle=\frac{1}{2 \pi j} \oint_{\Gamma} G(s) L_{k}(-s) d s=\operatorname{Res}\left(G(s) L_{k}(-s), s=p\right)
$$


The Laguerre coefficients of $F(s)=\sum_{n=0}^{\infty} f_{n} L_{n}$ are computed via

$$
f_{n}=\left[\frac{d^{n}}{d s^{n}} \frac{\sqrt{2 p}(s+p)^{n}}{n !} F(s)\right]_{s=p}
$$

\section{Derivation of Controller Design Equations}

In this section we derive the controller design equations. Assume

1. the desired controller is described by the as yet unknown transfer function $C(s)$ of order $\mathrm{n}$

2. the transfer function $P(s)$ is a good model of the plant

3. the transfer function $T(s)$ describes the desired closed loop behavior of the system

4. the relationship between the closed loop behavior, the plant, and the controller is given by $T(s)=P(s) C(s) /[1+P(s) C(s)]$

It is easy to "clear fractions" (leaving off the ubiquitous dependence on $s$ ) and collect terms involving $C$ on the left

$$
(1-T) P C=T
$$

The quantities $T,(1-T)$, and $P$, are rational functions with numerators and denominators so

$$
T=\frac{n_{T}}{d_{T}}, \quad 1-T=\frac{d_{T}-n_{T}}{d_{T}}, \quad P=\frac{n_{P}}{d_{P}}
$$

Substituting and "clearing fractions" again produces

$$
\left[d_{T}-n_{T}\right] n_{P} C=n_{T} d_{P} \Rightarrow \frac{\left[d_{T}-n_{T}\right] n_{P}}{(s+p)^{q}} C=\frac{n_{T} d_{P}}{(s+p)^{q}}
$$

Now substitute for $C$ to get

$$
\frac{\left[d_{T}-n_{T}\right] n_{p}}{(s+p)^{q}} \frac{n_{0} L_{0}+n_{1} L_{1}+\ldots+n_{r} L_{r}}{d_{0} L_{0}+d_{1} L_{1}+\ldots+d_{r} L_{r}}=\frac{n_{T} d_{p}}{(s+p)^{q}}
$$

almost done, partially "clear fractions" one more time to get

$$
\frac{\left[d_{T}-n_{T}\right] n_{P}}{(s+p)^{q}}\left[n_{0} L_{0}+n_{1} L_{1}+\ldots+n_{r} L_{r}\right]=\frac{n_{T} d_{P}}{(s+p)^{q}}\left[d_{0} L_{0}+d_{1} L_{1}+\ldots+d_{r} L_{r}\right]
$$

The final step is to take the inner product of both sides of this equation with $L_{i}$ for $i=0,1, \ldots$ to get the equations

$$
\left\langle\frac{\left[d_{T}-n_{T}\right] n_{P}}{(s+p)^{q}} \sum_{k=0}^{r} n_{k} L_{k}, L_{i}\right\rangle=\left\langle\frac{n_{T} d_{P}}{(s+p)^{q}} \sum_{k=0}^{r} d_{k} L_{k}, L_{i}\right\rangle
$$


These equations produce the unconstrained design equations in the form

$$
N n=D d \Rightarrow[-D \quad N] p=0
$$

where $p$ is the parameter vector consisting of the vector $d$ stacked on top of the vector $n$.

\section{Examples}

Simple illustrative examples are presented. Consider an unstable plant $P(s)=$ $\frac{1}{s-2}$. In this section, $p=1 / 2$ to minimize the decimal digits in numerical answers since $\sqrt{2 p}=1$. Also, $q$ is chosen to be as small as possible to ensure all expanded functions are strictly proper.

Zeroth order controller. $C=\frac{n_{0} L_{0}}{d_{0} L_{0}}=n_{0} / d_{0}=n_{0}$. With $d_{0}=1$ this is seen to be just a proportional controller. $C=\frac{n_{0} L_{0}}{d_{0} L_{0}}$. The monic constraint requires $d_{0}=1$ so $C=n_{0}$. The target transfer function is chosen to be $T=$ $3 /(s+2)$.

$$
\begin{gathered}
(1-T) P C=T \Rightarrow \frac{n_{1-T} n_{P}}{(s+p)^{q}}\left[n_{0}\right]=\frac{n_{T} d_{P}}{(s+p)^{q}} \\
\frac{s-1}{(s+p)^{q}} n_{0}=\frac{3(s-1)}{(s+p)^{q}}
\end{gathered}
$$

cancelling the $(s-1)$ and choosing $\mathrm{q}=1$

$$
\frac{1}{(s+p)} n_{0}=\frac{3}{(s+p)} \Rightarrow n_{0}=3
$$

This was a contrived example which had an exact answer. Suppose $T=$ $2 /(s+2)$ which cannot be exactly achieved with a $0^{\text {th }}$ order controller.

$$
\begin{gathered}
(1-T) P C=T \Rightarrow \frac{n_{1-T} n_{P}}{(s+p)^{q}}\left[n_{0}\right]=\frac{n_{T} d_{P}}{(s+p)^{q}} \\
(1-T) P C=T \Rightarrow \frac{s}{(s+0.5)^{2}}\left[n_{0}\right]=\frac{2(s-1)}{(s+0.5)^{2}}
\end{gathered}
$$

Choose $n_{0}$ to be the real value that minimizes the least squared error in the equations

$$
\begin{array}{rrrr}
\left\langle\frac{s}{(s+0.5)^{2}}\left[n_{0}\right], L_{i}\right\rangle & =\left\langle\frac{2(s-1)}{(s+0.5)^{2}}, L_{i}\right\rangle \\
i & \left\langle L H S, L_{i}\right\rangle & = & \left\langle R H S, L_{i}\right\rangle \\
i=0 & .5 n_{0} & = & -1 \\
i=1 & .5 n_{0} & = & 3
\end{array}
$$

so $n_{0}=2=C$ produces the closed loop transfer function $T=[2 /(s-1)] /[1+$ $2 /(s-1)]=2 /[s+1]$ 
First order controller. $C=\frac{n_{0} L_{0}+n_{1} L_{1}}{d_{0} L_{0}+d_{1} L_{1}}=\frac{n_{0}(s+p)+n_{1}(s-p)}{d_{0}(s+p)+d_{1}(s-p)}=$ $\frac{\left(n_{0}+n_{1}\right) s+p\left(n_{0}-n_{1}\right)}{\left(d_{0}+d_{1}\right) s+p\left(d_{0}-d_{1}\right)}$

The example continues with the unstable plant $P(s)=\frac{1}{s-1}$. There are two constraints on the denominator of the controller: a) the monic constraint, i.e. $d_{0}+d_{1}=1$ and the constraint that comes from requiring that the denominator of the controller be $s$ which guarantees zero steady state error do to a step input, this yields $d_{0}-d_{1}=0$. So $d_{0}=d_{1}=1 / 2$. Only the numerator coefficients remain to be determined. Simplifying

$$
(1-T) P C=T \Rightarrow \frac{n_{1-T} n_{P}}{(s+p)^{q}}\left[n_{0} L_{0}+n_{1} L_{1}\right]=\frac{n_{T} d_{P}}{(s+p)^{q}} \frac{L_{0}+L_{1}}{2}
$$

The target transfer function is chosen to be $T=\frac{2 s+1}{s^{2}+s+1}$.

$$
\frac{s^{2}-s}{(s+p)^{3}}\left[n_{0} L_{0}+n_{1} L_{1}\right]=\frac{(s-1)(2 s+1)}{(s+p)^{3}} \frac{L_{0}+L_{1}}{2}
$$

The equations for $n_{0}$ and $n_{1}$ are generated by taking the inner product of both sides this this equation (the Laguerre expansion coefficients were produced using Maple) with $L_{i}$ or $i=0,1,2,3,4, \ldots$

$$
\begin{array}{cccc}
i & \left\langle L H S, L_{i}\right\rangle & = & \left\langle R H S, L_{i}\right\rangle \\
i=0 & -0.25 n_{0} & = & -1 \\
i=1 & 0.75 n_{0}-0.25 n_{1} & = & 3 \\
i=2 & 0.25 n_{0}+.75 n_{1} & = & 1 \\
i=3 & -0.75 n_{0}+0.25 n_{1} & = & -3 \\
i=4 & -0.75 n_{1} & = & 0
\end{array}
$$

This is simply the matrix-vector equation $N n=b$ which can be solved $n=N^{+} b$ where the super script ${ }^{+}$indicates the Moore-Penrose pseudo inverse available in MatLab. Matlab produced $n_{0}=2$ and $n_{1}=0$ which produces the controller

$$
C(s)=\frac{n_{0} L_{0}+n_{1} L_{1}}{0.5 L_{0}+.5 L_{1}}=\frac{2(s+.5)+0(s-.5)}{0.5(s+.5)+0.5(s-.5)}=\frac{2 s+1}{s}
$$

The resulting closed loop transfer function is

$$
T=\frac{P C}{1+P C}=\frac{(2 s+1) /[s(s-1)]}{1+(2 s+1) /[s(s-1)]}=\frac{2 s+1}{s^{2}+s+1}
$$

Of course, this was a contrived example to demonstrate that the process works and to introduce the second constraint equation.

Now consider a target transfer function that is not exactly achievable, i.e. suppose the target transfer function is $T=\frac{s+1}{s^{2}+s+1}$. The design equations
are

$$
\left\langle n_{1-T} n_{P}\left(n_{0} L_{0}+n_{1} L_{1}\right), L_{i}\right\rangle=\left\langle n_{T} d_{P}\left(L_{0}+L_{1}\right) / 2, L_{i}\right\rangle
$$




$$
\begin{array}{cccc}
\left\langle\frac{s^{2}}{(s+.5)^{3}}\left(n_{0} L_{0}+n_{1} L_{1}\right), L_{i}\right\rangle=\left\langle\frac{(s+1(s-1)}{(x+.5)^{3}}\left(L_{0}+L_{1}\right) / 2, L_{i}\right\rangle \\
i & \left\langle L H S, L_{i}\right\rangle & = & \left\langle R H S, L_{i}\right\rangle \\
i=0 & -0.75 n_{0} & = & -0.625 \\
i=1 & 1.25 n_{0}-0.75 n_{1} & = & 1.75 \\
i=2 & 0.75 n_{0}+1.25 n_{1} & = & 1 \\
i=3 & -1.25 n_{0}+0.75 n_{1} & = & -1.75 \\
i=4 & -1.25 n_{1} & = & -0.375
\end{array}
$$

The best least squares solution produced by using the Moore-Penrose pseudo inverse (Matlab) is $n_{0}=1.334$ and $n_{1}=0.0810$. The controller is $C=$ $1.4151 s+0.6265$ in $\frac{s}{s}$ and the closed loop transfer function is $T=\frac{1.4151 s+0.6265}{s^{2}+0.4151 s+0.6265}$.

Second order controller. $C=\frac{n_{0} L_{0}+n_{1} L_{1}+n_{2} L_{2}}{d_{0} L_{0}+d_{1} L_{1}+d_{2} L_{2}}=$ $\frac{\left(n_{0}+n_{1}+n_{2}\right) s^{2}+2 p\left(n_{0}-n_{2}\right) s+p^{2}\left(n_{0}-n_{1}+n_{2}\right)}{\left(d_{0}+d_{1}+d_{2}\right) s^{2}+2 p\left(d_{0}-d_{2}\right) s+p^{2}\left(d_{0}-d_{1}+d_{2}\right)}$

Continuing with the same unstable plant $P=1 /(s-1)$ a second order controller will be designed to approximate the target transfer function $T=$ $\frac{s+1}{s^{2}+s+1}$. Recall $d_{0}+d_{1}+d_{2}=1$ imposes the monic constraint and $d_{0}-d_{1}+$ $d_{2}=0$ provides an integrator in the controller.

$$
\begin{gathered}
{\left[\begin{array}{cc}
1 & 1 \\
1 & -1
\end{array}\right]\left[\begin{array}{l}
d_{0} \\
d_{1}
\end{array}\right]=\left[\begin{array}{c}
1-d_{2} \\
-d_{2}
\end{array}\right] \Rightarrow\left[\begin{array}{l}
d_{0} \\
d_{1}
\end{array}\right]=\frac{1}{-2}\left[\begin{array}{cc}
-1 & -1 \\
-1 & 1
\end{array}\right]\left[\begin{array}{c}
1-d_{2} \\
-d_{2}
\end{array}\right]=\left[\begin{array}{c}
0.5-d_{2} \\
0.5
\end{array}\right]} \\
C=\frac{n_{0} L_{0}+n_{1} L_{1}+n_{2} L_{2}}{\left(0.5-d_{2}\right) L_{0}+0.5 L_{1}+d_{2} L_{2}}
\end{gathered}
$$

The design equations are generated from

$$
\frac{n_{1-T} n_{P}}{(s+.5)^{q}}\left[n_{0} L_{0}+n_{1} L_{1}+n_{2} L_{2}\right]=\frac{n_{T} d_{P}}{(s+p)^{q}}\left[\left(0.5-d_{2}\right) L_{0}+0.5 L_{1}+d_{2} L_{2}\right]
$$

or

$$
\begin{aligned}
& \frac{s^{2}}{(s+0.5)^{3}}\left[n_{0} L_{0}+n_{1} L_{1}+n_{2} L_{2}\right]=\frac{(s+1)(s-1)}{(s+0.5)^{3}}\left[\left(0.5-d_{2}\right) L_{0}+0.5 L_{1}+d_{2} L_{2}\right] \\
& i \quad\left\langle L H S, L_{i}\right\rangle \quad=\quad\left\langle R H S, L_{i}\right\rangle \\
& i=0 \quad .25 n_{0} \quad=-.375+.75 d_{2} \\
& i=1 \quad .25 n_{0}+.25 n_{1} \quad=1.25-3.25 d_{2} \\
& i=2-.25 n_{0}+.25 n_{1}+.25 n_{2}=2.5 d_{2} \\
& i=3-.25 n_{0}-.25 n_{1}+.25 n_{2}=-1.25+2.5 d_{2} \\
& i=4 \quad-.25 n_{1}-.25 n_{2}=.375-3.25 d_{2} \\
& i=5 \quad-.25 n_{2} \quad=\quad .75 d_{2}
\end{aligned}
$$


The resulting Laguerre coefficients are: $n_{0}=-.75, n_{1}=.25, n_{2}=-.75$ and $d_{0}=.25, d_{1}=.5, d_{2}=.25$. These produce the controller is $C=\frac{s^{2}-1}{s^{2}}$, and the resulting closed loop transfer function is $T=\frac{s+1}{s^{2}+s+1}$ which EXACTLY matches the target transfer function.

\section{Conclusions}

Given the transfer function of the plant and a target closed loop transfer function, a procedure has been developed to design the controller that produces the best match between the target closed loop transfer function that produced by the controller and $T=P C /[1+P C]$. The process uses the orthonormal set of s-domain Laguerre functions. As the order of the controller increases, the better the match becomes.

This paper should be viewed as the first in a series of at least 3 papers. This paper produced rational controllers for rational plants and rational target closed loop transfer functions. The next paper will address the problem of designing rational s-domain controllers for irrational s-domain plants (plants with time delays or perhaps fractional order derivatives) and a given s-domain rational closed loop transfer function. The third paper will address the problem of producing rational z-domain controllers for given rational or irrational s-domain plants and given rational z-domain target closed loop transfer functions.

\section{References}

[1] Heuberger, P. S. C., Van den Hof, P. M. J., Wahlberg, B., Eds. "Modelling and Identification with Rational Basis Functions", SpringerVerlag, London 2005.

[2] Olivier, P. System Identificaion Using Laguerre Basis Functions, TechRxiv. Preprint. https://doi.org/10.36227/techrxiv.13656701.v1

[3] Olivier, P. D. Approximate System Inverses, Electronic Letters, 1995, Vol. 31, N 23 pp 2050-2051.

\section{Philip D Olivier, PhD, PE:}

Dr. Olivier has earned a $\mathrm{PhD}$ in electrical engineering and an MS in mathematics from Texas Tech University and a BS in physics from Loyola University, New Orleans. He has taught electrical engineering at the University of South Alabama; Lawrence Technological University, Mercer University, The University of Texas at San Antonio, and Louisiana State University, Baton Rouge. Additionally he has held various administrative positions including Department Chair, Interim Assistant Dean, Assistant to the Dean, and Director of Funded 
Projects and the Honors Program. He is a Senior Member of IEEE. He can be reached via email at philip_d_olivier@ieee.org. 